\title{
Fast Exciton Dynamics and Coherent Oscillations Revealed by Coherent 2D Spectroscopy in Chlorosomes
}

\author{
J. Dostál ${ }^{1,2}$, T. Mančal ${ }^{2}$, F. Vácha ${ }^{3}$, R. Augulis ${ }^{1}$, J. Pšenčík $^{2}$, and D. Zigmantas ${ }^{1}$ \\ ${ }^{1}$ Department of Chemical Physics, Lund University, Getingevägen 60, 22100 Lund, Sweden \\ ${ }^{2}$ Faculty of Mathematics and Physics, Charles University in Prague, Ke Karlovu 3, 12116 Prague, \\ Czech Republic \\ ${ }^{3}$ Faculty of Science, University of South Bohemia, Branišovská 31, 37005 České Budějovice, Czech \\ Republic
}

\begin{abstract}
In this study ultrafast energy transfer dynamics in chlorosomes from sulphur bacterium Chlorobaculum tepidum were explored by means of coherent electronic twodimensional spectroscopy. Observed sub-100 fs dynamics were attributed to incoherent downhill excitation diffusion between disordered domains within chlorosomes. At the same time vibrational coherent oscillations were investigated on the longer timescales.
\end{abstract}

\section{Introduction}

Chlorosomes - light-harvesting antennae found in green photosynthetic bacteria - are in many respects unique among light-harvesting systems developed by nature. They are untypically large - up to $200 \mathrm{~nm}$ along their longest axes; very efficient - allowing the growth of some bacterial species under the extremely low light conditions hundreds of meters under water in the oceans. Chlorosome properties originate from the strong exciton coupling of a huge number $\left(\sim 10^{5}\right)$ of pigments (mainly bacteriochlorophylls) - rather than pigment-protein interactions as in the case of the other antennae [1].

Bacteriochlorophyll (BChl) molecules inside the chlorosome are arranged in the curved lamellar structures possibly forming multilayered cylinders. The arrangement is unique for every chlorosome. The distance between individual lamellar layers is given by the length of BChl's esterifying alcohol and was determined to be 2-3 $\mathrm{nm}$ by means of X-ray scattering and cryo-electron microscopy [2,3]. The detailed short-range arrangement of $\mathrm{BChl}$ molecules is still the matter of ongoing dispute.

Fast photophysical processes following an excitation of chlorosome $\mathrm{Q}_{\mathrm{y}}$ band were studied by several groups (for review see [1,4]). It was reported that initial fast exciton relaxation occurring in less than 1 ps is followed by the long-range energy equilibration inside the chlorosome. Later energy is transferred on the time scale of tens of picosecond to the baseplate - a pigment protein complex attached to the side of chlorosome oriented towards the cell membrane. Additionally, coherent oscillations attributed to ground-state vibrational coherences were also observed [5-7].

In this work we have studied chlorosomes from green sulphur bacterium Chlorobaculum tepidum by coherent two-dimensional (2D) optical spectroscopy at ambient and $80 \mathrm{~K}$ temperatures. 2D spectroscopy provides high simultaneous spectral and temporal resolution and thus is perfectly suited for studying fast energy transfer dynamics and coherent oscillations in multichromophoric systems. The details about experimental setup can be found in [8].

This is an Open Access article distributed under the terms of the Creative Commons Attribution License 2.0, which permits unrestricted use, distribution, and reproduction in any medium, provided the original work is properly cited. 

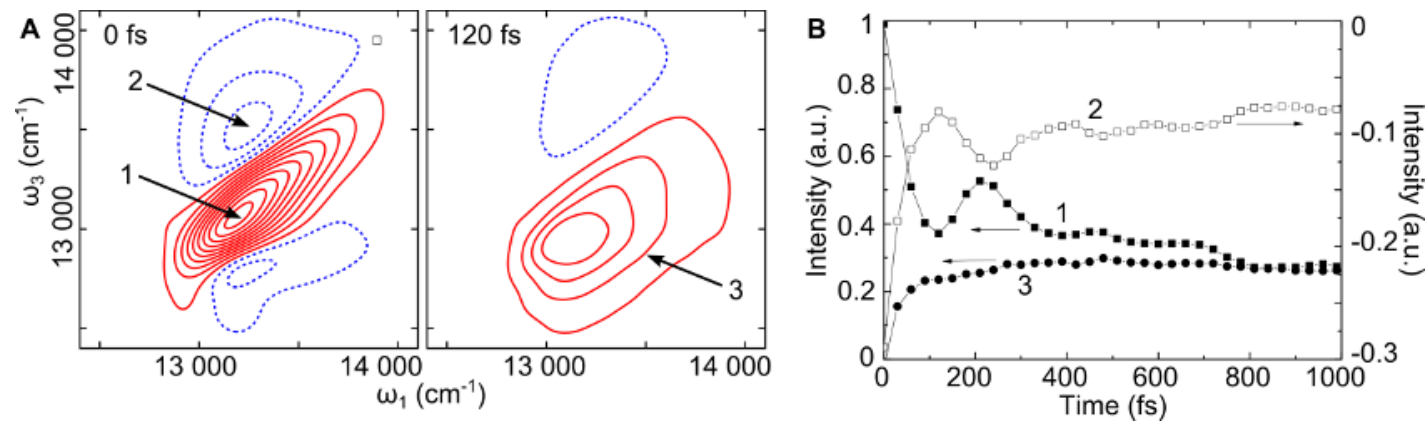

Fig. 1. (A) Measured 2D spectrum (the real part of electric field) of chlorosomes at $80 \mathrm{~K}$.

Positive contour lines are solid, negative are dashed. (B) Time evolution of the $2 \mathrm{D}$ spectrum in different areas as pointed by the arrows in A.

\section{Results and Discussion}

2D spectra of chlorosomes were measured at both ambient and $80 \mathrm{~K}$ temperatures. 2D spectra sequences recorded at both temperatures exhibit the same general features (Fig. 1.):

- Extensive elongation of the positive part - corresponding to stimulated emission (SE) and ground state bleaching (GSB) - in the diagonal direction at the time immediately after excitation. Below and above this signal there is negative excited state absorption (ESA) signal. The elongation indicates significant disorder in the observed system. As single molecular spectroscopy measurements shows negligible differences between individual chlorosomes [9], this disorder has to be present inside every chlorosome.

Therefore we propose that due to small random irregularities of $\mathrm{BChl}$ arrangement the chlorosomal aggregate is effectively broken into coherent domains constituted of small group of excitonicaly coupled pigments. Individual coherent domains are only weakly coupled to their neighbours. The elongated shape is thus a manifestation of the broad energy distribution of the position of a single exciton band located in different coherent domains.

- Fast initial evolution. During the first $100 \mathrm{fs}$ after excitation the elongated shape of the positive signal becomes significantly rounded. This process is accompanied by the decrease of signal intensity that drops to less than half of its initial value with the effective time constant shorter than $50 \mathrm{fs}$. The diagonal direction of nodal line separating positive and negative signal remains without appreciable change.

This behaviour is interpreted in terms of proposed coherent domain model as downhillbiased diffusion between neighbouring coherent domains [10]. Random energy transfer from high energy domains to their lower-lying neighbours results in filling the area under diagonal. Positive part of spectrum thus becomes rounder with diagonal nodal line.

- Coherent oscillations were observed with main frequencies of approximately 90 and 145 $\mathrm{cm}^{-1}$ damped during $1.2 \mathrm{ps}$ time at $80 \mathrm{~K}$ temperature. The oscillating pattern is almost identical everywhere in the 2D spectrum. There are differences only in the amplitude, which varies (including its sign) from one point to another in the $2 \mathrm{D}$ spectrum. Interestingly, the distribution of the oscillating-pattern amplitude at any time of the system evolution resembles the shape of the 2D spectrum at $0 \mathrm{fs}$ and is not changing according to the actual 2D spectrum given by the ultrafast diffusion process (Fig. 2). The oscillating behaviour is more pronounced at low temperature conditions, but is observed and ambient temperature as well.

Similar oscillations were attributed to the ground state vibrational coherence $[5,6]$. However, presence of the oscillations with negative amplitude in the ESA part of the 2D spectra indicates that at least part of the oscillating signal comes from the excited state 
coherences. The distribution of the oscillations in the 2D spectrum mimicking its initial shape suggests that both excited and ground state coherences are affected during the ultrafast diffusion process and thus we observe only coherences from those domains that do not transfer energy on sub-100 fs timescale.

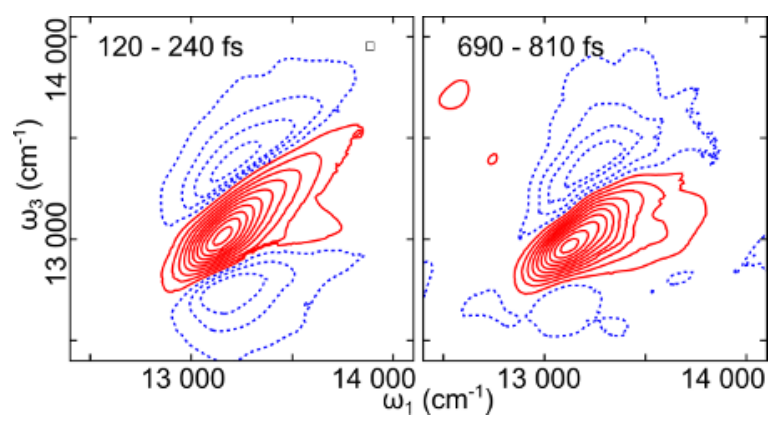

Fig. 2. Distribution of the oscillatory pattern amplitude (determined by the signal intensity difference at times of two consecutive oscillation extremes) at different population time.

Positive contour lines are solid, negative are dashed.

\section{Conclusions}

We have explored ultrafast excitation energy dynamics in chlorosomes caused by the downhillbiased exciton diffusion between the coherent domains. Effective stochastic diffusion assures efficient energy transfer through chlorosomes at the earlier time after the excitation. We also observed clear vibrational wavepacket dynamics on the ground and excited electronic states.

\section{References}

1. R. E. Blankenship, K. Matsuura in, Light-harvesting antennas in photosynthesis, B. R. Green, W. W. Parson ed. (Kluwer Academic Publishers, Dordrecht; 2003)

2. J. Psencik, T. P. Ikonen, P. Laurinmaki, M. C. Merckel, S. J. Butcher, R. E. Serimaa, et al., Biophys J 87, 1165 (2004)

3. G. T. Oostergetel, M. Reus, A. G. M. Chew, D. A. Bryant, E. J. Boekema, A. R. Holzwarth, FEBS Lett 581, 5435 (2007)

4. G. T. Oostergetel, H. Van Amerongen, E. J. Boekema, Photosynth Res 104, 245 (2010)

5. Y. Z. Ma, J. Aschenbrucker, M. Miller, T. Gillbro, Chem Phys Lett 300, 465 (1999)

6. V. I. Prokhorenko, D. B. Steensgaard, A. R. Holzwarth, Biophys J 79, 2105 (2000)

7. S. Savikhin, P. I. Vannoort, Y. W. Zhu, S. Lin, R. E. Blankenship, W. S. Struve, Chem Phys 194, 245 (1995)

8. R. Augulis, D. Zigmantas, Opt Express 19, 13126 (2011)

9. Y. Tian, R. Camacho, D. Thomsson, M. Reus, A. R. Holzwarth, I. G. Scheblykin, Journal of the American Chemical Society 133, 17192 (2011)

10. J. Dostal, T. Mancal, F. Vacha, R. Augulis, J. Psencik, D. Zigmantas, Journal of the American Chemical Society, doi: 10.1021/ja3025627 (2012) 